\title{
Estimation of parameters in the Information Based Asset Pricing Framework
}

\author{
Cynthia Ikamari ${ }^{1,2}$, Philip Ngare $^{2}$ and Patrick Weke ${ }^{2}$ \\ 1. Faculty of Business and Economics, Multimedia University of Kenya, Kenya \\ 2. School of Mathematics, University of Nairobi, Kenya
}

\begin{abstract}
Stochastic volatility models have become common in pricing options due to their ability to capture the changes in the asset's volatility with time. The information based asset pricing framework proposed by Brody, Hughson and Macrina referred to the BHM model or BHM approach is an improvement to the existing stochastic volatility models as it incorporates information in determining the option price dynamics. The objective of this study is to extend the application of a non-linear filtering approach used in volatility extraction in the Heston model to the BHM model. The measurement and transition equations obtained in the state space model are used in the extended kalman filter to extract volatility. The BHM model from a Black-Scholes perspective updated in the results of the Gaussian Integrals is referred to the BS-BHM Updated model. The option price is obtained using this model and the parameters which cannot be observed directly in the model are estimated using the method of moments.
\end{abstract}

Keywords: BHM model, BS-BHM Updated model, method of moments, stochastic volatility.

DOI: $10.7176 / \mathrm{MTM} / 9-5-08$

Publication date: May 31st 2019

Correspondence should be addressed to Cynthia Ikamari; cynikamari@gmail.com 


\section{Introduction}

The Black Scholes model as proposed in the landmark paper by Black and Scholes(1973) is a fundamental model in derivatives pricing. A derivative is a financial contract whose value depends on an underlying asset such as stock. An option is a financial derivative contract which gives the holder the right but not the obligation to buy or sell a particular asset at an agreed price at a specified future date. A European call option gives the holder the right but not the obligation to buy a particular asset at a stated price at a specified future date.

The assumption of constant volatility in the Black Scholes model has been shown by empirical studies not to match the economical phenomenon in reality. It is found that for call options in particular, the changes in volatility for the strike prices vary significantly as compared to the spot price of the asset, this feature is referred to as the volatility smile. The Black Scholes model therefore does not capture the volatility smile.

To address this weakness of the Black Scholes model, stochastic volatility models(SVMs) were developed in the 1980s when Hull and White(1987) and others generalized the model. SVMs are able to capture the volatility smile by allowing the volatility of the underlying asset to evolve according to its own stochastic dynamics. They are useful because they explain in a consistent way why call options with different strikes and time to maturity have different Black Scholes implied volatilities.

The Heston model as introduced in Heston(1993) is a popular stochastic volatility model used in derivative pricing. Its variance process follows a version of the square root process described by Cox, Ingersoll and Ross(1985). The model offers a practical method to account for stochastic volatility as a closed form solution to the model exists and is therefore seen as an improvement to the Black Scholes model.

However, the Heston model suffers from the weakness of specifying on an ad hoc basis the volatility dynamics. The information based asset pricing framework as proposed by Brody, Hughson and Macrina(BHM) addresses this weakness by generating a natural explanation for the origin of stochastic volatility in financial markets without the need for the dynamics of the volatliity process being specified on an ad hoc basis.

In the BHM model, the concept of noisy information about the future cash flows is used to derive the corresponding price processes. In this framework, an asset is defined by its corresponding cash flow structure. Each cash flow is modelled by a random variable which can be associated to a market information process consisting of a sum of two terms: one containing the noisy information and the other containing the true information about the value of the linked market factor.

In their paper, Brody et al(2008) show that the Black Scholes model can be derived from an information based perspective, this model is referred to the BS-BHM model. Later, Mutijah et al(2013) updated the model in the results of the Guassian integrals, specifically in the analysis of the algebra trick of completing square. The updated Black Scholes model from an information based framework is referred to as the BS-BHM Updated model.

In this study, the focus is on the estimation of parameters used in determining the price of a European call option under the BS-BHM Updated model. The approach to estimation used will be based on the method of moments. Hall(2005) shows that the method involves matching the moment of the population with the empirical moments so as to obtain estimators of the parameters of interest. The parameter estimators are 
obtained by solving a system of equations of the form:

$$
\begin{aligned}
\mu_{j} & =\mathbb{E}\left[X^{j}\right] \\
& =\frac{1}{n} \sum_{i=1}^{n} X_{i}^{j}, \quad j=1, \ldots, k
\end{aligned}
$$

where $k$ denotes the number of parameters to be estimated.

In the BS-BHM Updated model, two parameters will be estimated: the volatility parameter, $v$ and the information flow rate parameter, $\sigma$ which cannot be directly observed. This study looks at the estimation of these two parameters using the method of moments.

This work is not the first attempt in estimation of parameters in the BS-BHM Updated model, Mutijah et al (2013) have previously looked at the estimation of parameters in the BS-BHM Updated model using Monte Carlo Estimation and the Method of Moments. This study attempts to update the result obtained by Mutijah et al(2013) using the method of moments. In particular, the equation of the population moments to the empirical moments in the method of moments is updated and a different result is obtained.

The study starts by reviewing the BHM model in section 2 which derives the asset price dynamics by modelling the market information. Information is then incorporated to the Black Scholes model to obtain the BS-BHM model which is later updated by Mutijah(2013) to obtain the BS-BHM Updated model. The price of the European call option under the BS-BHM Updated model is obtained under the risk neutral measure.

In section 3, the method of moments is applied to the BS-BHM Updated model and two estimators are obtained for the volatility parameter and the true information flow rate. Numerical results are then given in section 4 followed by a conclusion in section 5 .

\section{The BHM Model}

The BHM model as presented in Brody, Hughson and Macrina(2008) views asset price movements as an emergent phenomenon. The model's basis is pricing of assets by modelling the flow of market information. The market information in this case relates to the given assets expected future cash flows. The BHM approach is different from the other methods used in pricing assets mainly because the stochastic process governing the underlying asset price dynamics is deduced rather than being imposed in an arbitrary way.

The BHM approach is first studied where the Brownian motion that drives the price process is obtained. The asset price dynamics from the BHM approach are then used to determine the asset price dynamics in the BS-BHM Updated model. The BS-BHMUpdated model as presented by Mutijah, et. al(2012) uses Gaussian integrals to determine the equation of the asset pricing model. The result obtained is different from that of BS-BHM model due to an imprecision made in the Gaussian Integrals by BHM.

Consider a single cash flow occurring at time $T$, represented by a random variable, $X_{T}$. The value of this variable will be revealed at time $T$. The flow of market information available to market participants is assumed to be contained in a process $\left\{\xi_{t}\right\}_{0 \leq t \leq T}$ given by:

$$
\xi_{t}=\sigma t X_{t}+\beta_{t T}
$$

$\xi_{t}$ denotes the market information process, its also known as the information process. $\sigma t X_{T}$ contains the 'true information' about the value of the cash flow $X_{T} . \sigma$ denotes the 
rate at which the true value of $X_{T}$ is revealed to the market participants. $\left\{\beta_{t T}\right\}_{0 \leq t \leq T}$ denotes a standard Brownian bridge over the interval $[0, T]$ with $\beta_{0 T}=0$ and $\beta_{T T}=0$.

$$
\beta_{t T} \sim N\left(0, \frac{t(T-t)}{T}\right)
$$

$X_{T}$ and $\beta_{t T}$ are assumed to be independent in the information-based framework.

From the market's point of view, it is the process $W_{t}$ that drives the asset price dynamics. According to Brody, Hughson and Macrina(2008), the dynamics of the price process are given as:

$$
d S_{t}=r_{t} S_{t} d t+\Gamma_{t T} d W_{t}
$$

In this study, an assumption will be made that $r_{t}$ is a constant which implies that $r_{t}=r$. Thus;

$$
d S_{t}=r S_{t} d t+\Gamma_{t T} d W_{t}
$$

where $\Gamma_{t T}$ denotes the absolute volatility process:

$$
\Gamma_{t T}=P_{t T} \frac{\sigma T}{T-t} V_{t}
$$

$P_{t T}$ denotes the discount factor and $r_{t}$ denotes the short rate.

By using Gaussian integrals, the equation of asset pricing model is presented as in Mutijah, et. al(2012) in the BS-BHM-Updated model as follows:

$$
S_{t}=S_{0} \exp \left(r t-\frac{1}{2} \frac{\sigma^{2} \tau}{\sigma^{2} \tau+1} v^{2} T+\frac{\sigma \tau v \sqrt{T}}{t\left(\sigma^{2} \tau+1\right)} \xi_{t}\right)
$$

where $\tau=\frac{t T}{T-t}$

$v$ is the asset price volatility parameter. $\sigma$ and $v$ cannot be observed directly and are the subject of estimation in this work. The equation above gives a different final result as compared to that of BHM's Black-Scholes Model from an Information-Based Perspective;

$$
S_{t}=P_{t T} S_{0} \exp \left(r T-\frac{1}{2} v^{2} T+\frac{1}{2} \frac{v \sqrt{T}}{\sigma^{2} \tau+1}+\frac{\sigma \tau v \sqrt{T}}{t\left(\sigma^{2} \tau+1\right)} \xi_{t}\right)
$$

The difference arises due to an imprecision done by BHM using Gaussian Integrals.

Mutijah et al(2013) show that the BS-BHM Updated model in equation 2.4 follows a lognormal distribution:

$$
\begin{gathered}
\frac{S_{t}}{S_{0}}=\exp \left(r T-\frac{1}{2} v^{2} T+\frac{1}{2} \frac{v \sqrt{T}}{\sigma^{2} \tau+1}+\frac{\sigma \tau v \sqrt{T}}{t\left(\sigma^{2} \tau+1\right)} \xi_{t}\right) \\
\log \left(\frac{S_{t}}{S_{0}}\right)=r T-\frac{1}{2} v^{2} T+\frac{1}{2} \frac{v \sqrt{T}}{\sigma^{2} \tau+1}+\frac{\sigma \tau v \sqrt{T}}{t\left(\sigma^{2} \tau+1\right)} \xi_{t} \\
\log S_{t} \sim N\left(\log S_{0}+r t-\frac{1}{2} \frac{\sigma^{2} \tau}{\sigma^{2} \tau+1} v^{2} T,\left(\frac{\sigma \tau v \sqrt{T}}{t\left(\sigma^{2} \tau+1\right)}\right)^{2}\left(\sigma^{2} t^{2}+\frac{t(T-t)}{T}\right)\right) \\
E\left[S_{t}\right]=S_{0} \exp \left(r t-\frac{1}{2} \frac{\sigma^{2} \tau}{\sigma^{2} \tau+1} v^{2} T\right) \\
\operatorname{Var}\left[S_{t}\right]=S_{0}^{2}\left(\exp 2\left(r t-\frac{1}{2} \frac{\sigma^{2} \tau}{\sigma^{2} \tau+1} v^{2} T\right)\right)\left(\exp \left(\left(\frac{\sigma \tau v \sqrt{T}}{t\left(\sigma^{2} \tau+1\right)}\right)^{2}\right)\left(\sigma^{2} t^{2}+\frac{t(T-t)}{T}\right)-1\right)
\end{gathered}
$$


Thus;

$$
\begin{gathered}
E\left[\log \left(\frac{S_{t}}{S_{0}}\right)\right]=r t-\frac{1}{2} \frac{\sigma^{2} \tau}{\sigma^{2} \tau+1} v^{2} T \\
\operatorname{Var}\left[\log \left(\frac{S_{t}}{S_{0}}\right)\right]=\left(\frac{\sigma \tau v \sqrt{T}}{t\left(\sigma^{2} \tau+1\right)}\right)^{2}\left(\sigma^{2} t^{2}+\frac{t(T-t)}{T}\right)
\end{gathered}
$$

$$
\log \left(\frac{S_{t}}{S_{0}}\right) \sim N\left[r t-\frac{1}{2} \frac{\sigma^{2} \tau}{\sigma^{2} \tau+1} v^{2} T,\left(\frac{\sigma \tau v \sqrt{T}}{t\left(\sigma^{2} \tau+1\right)}\right)^{2}\left(\sigma^{2} t^{2}+\frac{t(T-t)}{T}\right)\right]
$$

Let $A=r t-\frac{1}{2} \frac{\sigma^{2} \tau}{\sigma^{2} T+1} v^{2} T$ and $B^{2}=\left(\frac{\sigma \tau v \sqrt{T}}{\tau\left(\sigma^{2} T+1\right)}\right)^{2}\left(\sigma^{2} t^{2}+\frac{t(T-t)}{T}\right)$.

This implies that

$$
\begin{gathered}
\log \left(\frac{S_{t}}{S_{0}}\right) \sim N\left[A, B^{2}\right] \\
S_{t} \sim N\left[S_{0} e^{A}, S_{0}^{2} e^{2 A}\left(e^{B^{2}}-1\right)\right] \\
S_{t}=S_{0} e^{A+B Z}
\end{gathered}
$$

where $\mathrm{Z}$ denotes a standard normal random variable.

The European call option price under the BS-BHM-Updated model at time $t$ with expiration date $\mathrm{T}$ and strike price $\mathrm{K}$ takes the form

$$
C=E_{\mathrm{Q}}\left[\max \left\{S_{t}-K, 0\right\}\right]
$$

where $\mathbb{Q}$ is the risk neutral probability and $\mathrm{C}$ denotes the European call price. Following a similar approach to Macrina (2006), the existence of an established pricing kernel and the absence of arbitrage is assumed. These two assumptions ensure the existence of a unique risk neutral probability measure $\mathbb{Q}$. In addition, an assumption of a default-free deterministic interest rate is made.

$$
\begin{array}{r}
C=\int_{0}^{\infty} \max \left\{S_{t}-K, 0\right\} f\left(s_{t}\right) d s_{t} \\
=\int_{K}^{\infty}\left\{S_{t}-K, 0\right\} f\left(s_{t}\right) d s_{t}
\end{array}
$$

Using the result from equation 2.8 and making $\mathrm{Z}$ to be the subject;

$$
Z=\frac{\log \left(\frac{S_{r}}{S_{0}}\right)-A}{B}
$$

Thus, for the lower limit, when $S_{t}=K$;

$$
\begin{gathered}
Z=\frac{\log \left(\frac{K}{S_{0}}\right)-A}{B} \\
C=\int_{\frac{\log \left(\frac{K}{S_{0}}\right)_{-A}}{B}}^{\infty}\left(S_{0} e^{A+B Z}-K\right) f(z) d z \\
=\int_{\frac{\log \left(\frac{K}{S_{0}}\right)_{-A}}{B}}^{\infty} S_{0} e^{A+B Z} f(z) d z-\int_{\frac{\log \left(\frac{K}{S_{0}}\right)_{-A}}{B}}^{\infty} K f(z) d z \\
=\int_{\frac{\log \left(\frac{K}{S_{0}}\right)_{-A}}{B}}^{\infty} S_{0} e^{A+B Z} f(z) d z-K \int_{\frac{\log \left(\frac{K}{S_{0}}\right)_{-A}}{B}}^{\infty} f(z) d z
\end{gathered}
$$




$$
\begin{aligned}
& f(z)=\frac{1}{\sqrt{2 \pi}} e^{-\frac{z^{2}}{2}} \\
& C=S_{0} e^{A} \int_{\frac{\log \left(\frac{K}{3_{0}}\right)_{-A}}{B}}^{\infty} e^{B Z} \frac{1}{\sqrt{2 \pi}} e^{-\frac{z^{2}}{2}} d z-K \int_{\frac{\log \left(\frac{K}{s_{0}}\right)_{-A}}{B}}^{\infty} \frac{1}{\sqrt{2 \pi}} e^{-\frac{z^{2}}{2}} d z \\
& =S_{0} e^{A} \int_{\frac{\log \left(\frac{K}{s_{B}}\right)_{-A}}{B}}^{\infty} e^{B Z-\frac{z^{2}}{2}} \frac{1}{\sqrt{2 \pi}} d z-K \int_{\frac{\log \left(\frac{K}{s_{B}}\right)_{-A}}{B}}^{\infty} \frac{1}{\sqrt{2 \pi}} e^{-\frac{z^{2}}{2}} d z \\
& B Z-\frac{z^{2}}{2}=\frac{B^{2}}{2}-\frac{(z-B)^{2}}{2} \\
& C=S_{0} e^{A} \int_{\frac{\log \left(\frac{K}{s_{0}}\right)_{-A}}{B}}^{\infty} e^{\frac{B^{2}}{2}-\frac{(z-B)^{2}}{2}} \frac{1}{\sqrt{2 \pi}} d z-K \int_{\frac{\log \left(\frac{K}{S_{0}}\right)_{-A}}{B}}^{\infty} \frac{1}{\sqrt{2 \pi}} e^{-\frac{z^{2}}{2}} d z \\
& =S_{0} e^{A+\frac{B^{2}}{2}} \int_{\frac{\log \left(\frac{K}{S_{0}}\right)_{-A}}{B}}^{\infty} e^{-\frac{(z-B)^{2}}{2}} \frac{1}{\sqrt{2 \pi}} d z-K \int_{\frac{\log \left(\frac{K}{S_{0}}\right)_{-A}}{B}}^{\infty} \frac{1}{\sqrt{2 \pi}} e^{-\frac{z^{2}}{2}} d z \\
& =S_{0} e^{A+\frac{B^{2}}{2}} P\left[N(B, 1)>\frac{\log \left(\frac{K}{S_{0}}\right)-A}{B}\right]-K P\left[N(0,1)>\frac{\log \left(\frac{K}{S_{0}}\right)-A}{B}\right] \\
& =S_{0} e^{A+\frac{B^{2}}{2}}\left(1-\Phi\left(\frac{\log \left(\frac{K}{S_{0}}\right)-A}{B}-B\right)\right)-K\left(1-\Phi\left(\frac{\log \left(\frac{K}{S_{0}}\right)-A}{B}\right)\right) \\
& =S_{0} e^{A+\frac{B^{2}}{2}} \Phi\left(B-\frac{\log \left(\frac{K}{S_{0}}\right)-A}{B}\right)-K \Phi\left(\frac{A-\log \left(\frac{K}{S_{0}}\right)}{B}\right) \\
& =S_{0} e^{A+\frac{B^{2}}{2}} \Phi\left(\frac{\log \left(\frac{S_{0}}{K}\right)+A}{B}+B\right)-K \Phi\left(\frac{\log \left(\frac{S_{0}}{K}\right)+A}{B}\right) \\
& =S_{0} \Phi\left(\frac{\log \left(\frac{S_{0}}{K}\right)+A}{B}+B\right)-K e^{-\left(A+\frac{B^{2}}{2}\right)} \Phi\left(\frac{\log \left(\frac{S_{0}}{K}\right)+A}{B}\right)
\end{aligned}
$$

Let $d_{1}=\frac{\log \left(\frac{s_{0}}{K}\right)+A}{B}+B$ and $d_{2}=d_{1}-B$

$$
\phi(x)=\frac{1}{\sqrt{2 \pi}} \int_{-\infty}^{x} e^{-\frac{u^{2}}{2}} d u
$$

Thus, the information based asset pricing formula takes a similar form to the BlackScholes Model:

$$
C=S_{0} \Phi\left(d_{1}\right)-K e^{-\left(A+\frac{B^{2}}{2}\right)} \Phi\left(d_{2}\right)
$$

From equation 2.7 , the random variable $\left(\frac{S_{\mathrm{r}}}{S_{0}}\right)$ has a lognormal distribution with a density function given as follows:

$$
f\left(\frac{S_{t}}{S_{0}}\right)=\frac{1}{B \sqrt{2 \pi}\left(\frac{S_{t}}{S_{0}}\right)} \exp \left(-\frac{1}{2}\left[\frac{\log \left(\frac{S_{t}}{S_{0}}\right)-A}{B}\right]^{2}\right)
$$




\section{The Method of Moments}

Suppose that $S_{t_{1}}$ denotes the historical asset price at time $t_{1}$.

Let

$$
U_{i}=\log \left[\frac{S_{t_{i}}}{S_{t_{1-1}}}\right]
$$

Following the approach by Higham(2004), the $U_{i}$ 's are assumed to be independent of each other and denote the log of the ratio of the underlying asset prices.

Given that $t_{n}$ denotes the current time, $S_{t_{n}}$ denotes the current asset price and the asset prices for the most recent $M+1$ time periods are given as:

$$
\left(S_{t_{n-M}}, S_{t_{n-M+1}}, \ldots, S_{t_{n-1}}, S_{t_{n}}\right)
$$

The corresponding asset log ratio data will be given as:

$$
\left(U_{t_{n-M}}, U_{t_{n-M+1}}, \ldots, U_{t_{n-1}}, U_{t_{n}}\right)
$$

The first sample mean which denotes the sample mean is given as:

$$
m_{1}=\frac{1}{m} \sum_{n=1}^{M+1} U_{n+1-i}
$$

Similarly, the second sample moment is given as:

$$
m_{2}=\frac{1}{m} \sum_{n=1}^{M+1}\left(U_{n+1-i}\right)^{2}
$$

Suppose that $\mu_{1}$ and $\mu_{2}$ denote the first and second moments respectively for the BSBHM Updated model. It follows that:

$$
\begin{aligned}
\mu_{1} & =E\left[\mu_{i}\right] \\
& =\frac{1}{2} \frac{\sigma^{2} \tau}{\sigma^{2} \tau+1} v^{2} T
\end{aligned}
$$

and

$$
\begin{aligned}
\mu_{2} & =E\left[\mu_{i}^{2}\right] \\
& =\operatorname{Var}\left[\mu_{i}\right]+\left(E\left[\mu_{i}\right]\right)^{2} \\
& =\left(\frac{\sigma \tau v \sqrt{T}}{t\left(\sigma^{2} \tau+1\right)}\right)^{2}\left(\sigma^{2} t^{2}+\frac{t(T-t)}{T}\right)+\left(\frac{1}{2} \frac{\sigma^{2} \tau}{\sigma^{2} \tau+1} v^{2} T\right)^{2}
\end{aligned}
$$

The next step is to equate the sample moments to the moments from the model as follows:

$$
\begin{aligned}
m_{1} & =\mu_{1} \\
& =\frac{1}{2} \frac{\sigma^{2} \tau}{\sigma^{2} \tau+1} v^{2} T
\end{aligned}
$$

Making $v^{2}$ to be the subject:

$$
\begin{aligned}
v^{2} & =\frac{2\left(r t-m_{1}\right)\left(\sigma^{2} \tau+1\right)}{\sigma^{2} \tau T} \\
& =\frac{2\left(r t-m_{1}\right)}{T}+\frac{2\left(r t-m_{1}\right)}{\sigma^{2} \tau T}
\end{aligned}
$$


Similarly:

$$
\begin{aligned}
m_{2} & =\mu_{2} \\
& =\left(\frac{\sigma \tau v \sqrt{T}}{t\left(\sigma^{2} \tau+1\right)}\right)^{2}\left(\sigma^{2} t^{2}+\frac{t(T-t)}{T}\right)+\left(\frac{1}{2} \frac{\sigma^{2} \tau}{\sigma^{2} \tau+1} v^{2} T\right)^{2} \\
v^{2} & =\frac{\left(m_{2}-m_{1}^{2}\right) t\left(\sigma^{2} \tau+1\right)^{2}}{\sigma^{2} \tau^{2}\left(\sigma^{2} t T+(T-t)\right)}
\end{aligned}
$$

Equating equation 3.8 and equation 3.10 yields:

$$
\begin{aligned}
\frac{2\left(r t-m_{1}\right)\left(\sigma^{2} \tau+1\right)}{\sigma^{2} \tau T} & =\frac{\left(m_{2}-m_{1}^{2}\right) t\left(\sigma^{2} \tau+1\right)^{2}}{\sigma^{2} \tau^{2}\left(\sigma^{2} t T+(T-t)\right)} \\
\frac{2\left(r t-m_{1}\right)}{T} & =\frac{\left(m_{2}-m_{1}^{2}\right) t\left(\sigma^{2} \tau+1\right)}{\tau\left(\sigma^{2} t T+(T-t)\right)}
\end{aligned}
$$

Making $\sigma^{2}$ to be the subject:

$$
\begin{aligned}
2 \tau\left(r t-m_{1}\right)\left(\sigma^{2} t T+(T-t)\right) & =t T\left(m_{2}-m_{1}^{2}\right)\left(\sigma^{2} \tau+1\right) \\
2 \tau \sigma^{2} t T\left(r t-m_{1}\right)+2 \tau\left(r t-m_{1}\right)(T-t) & =t T \sigma^{2} \tau\left(m_{2}-m_{1}^{2}\right)+t T\left(m_{2}-m_{1}^{2}\right) \\
\sigma^{2} & =\frac{t T\left(m_{2}-m_{1}^{2}\right)+2 \tau\left(r t-m_{1}\right)(T-t)}{\left(2 \tau t T\left(r t-m_{1}\right)-t T \tau\left(m_{2}-m_{1}^{2}\right)\right)}
\end{aligned}
$$

Thus:

$$
\hat{\sigma}=\sqrt{\frac{t T\left(m_{2}-m_{1}^{2}\right)+2 \tau\left(r t-m_{1}\right)(T-t)}{\left(2 \tau t T\left(r t-m_{1}\right)-t T \tau\left(m_{2}-m_{1}^{2}\right)\right)}}
$$

Substituting equation (3.12) into equation (3.9), an estimate for $v$ can be obtained as follows:

$$
v^{2}=\frac{2\left(r t-m_{1}\right)}{T}+\frac{2\left(r t-m_{1}\right)\left[2 t\left(r t-m_{1}\right)-t\left(m_{2}-m_{1}^{2}\right)\right]}{t T\left(m_{2}-m_{1}^{2}\right)+2 \tau\left(r t-m_{1}\right)(T-t)}
$$

Thus:

$$
\hat{v}=\sqrt{\frac{2\left(r t-m_{1}\right)}{T}+\frac{2\left(r t-m_{1}\right)\left[2 t\left(r t-m_{1}\right)-t\left(m_{2}-m_{1}^{2}\right)\right]}{t T\left(m_{2}-m_{1}^{2}\right)+2 \tau\left(r t-m_{1}\right)(T-t)}}
$$

\section{Numerical Results}

The data used in this study relates to SPX call options daily data obtained from yahoo finance. The data covers the period from 17th February 2019 with a maturity date of 17th May 2019 with a total of 53 observations. The summary of the data is given in table 1.

\begin{tabular}{ccc}
\hline \hline Strike & Last Price & Log Ratio \\
\hline Min. : 2300 & Min. : 0.01 & Min. : 0.670 \\
1stQu. : 2705 & 1stQu. : 33.53 & 1stQu. : 1.090 \\
Median : 2830 & Median : 101.70 & Median : 1.450 \\
Mean : 2808 & Mean : 139.07 & Mean : 1.806 \\
3rdQu. : 2920 & 3rdQu. : 218.60 & 3rdQu. : 1.940 \\
Max. : 3300 & Max. : 486.12 & Max. : 5.520 \\
\hline
\end{tabular}

Table 1: SPX Call Options Data Summary 
The method of moments is used to estimate the volatility parameter, $v$ and the information flow rate, $\sigma$ of SPX call options for daily data. An assumption is made that the data corresponds to equally spaced points in time.

Estimation using the method of moments produces an estimate for the volatility parameter, $\hat{v}=0.005047805$ and the true information flow rate, $\hat{\sigma}=0.07104789$.

Figure 1 shows the increase in the rate of the information flow rate parameter as time increases. This is due to the fact that as the maturity period of 91 days approaches, the rate at which information about the underlying asset is revealed increases.

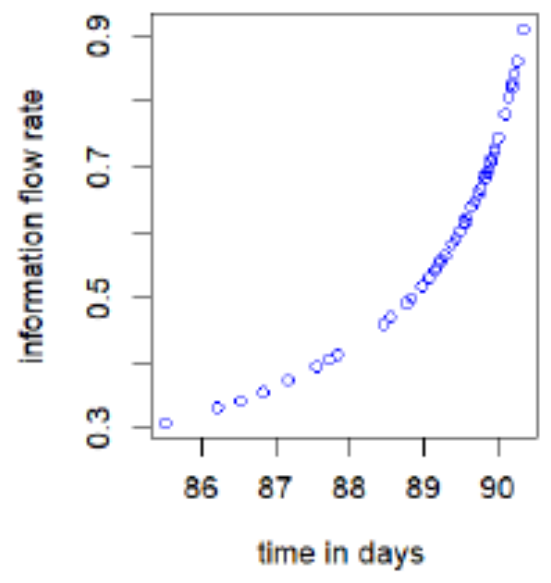

Figure 1: Information flow rate increase with time

\section{Conclusion}

The BS-BHM Updated model is an extension of the BS-BHM model as presented by Brody Hughson and Macrina where infromation is incorporated in the Black Scholes model. The price of a European call option is derived based on this model. The volatility parameter and information flow rate parameter which as required to determine the price cannot be observed directly from the data.

This study finds an estimate for these two parameters using the method of moments. A different result is obtained as compared to that obtained by Mutijah et al(2013) for the equation used to estimate the volatility parameter and the information flow rate parameter. This is as a result of an imprecision made in equating the population moments to the sample moments.

The values of these two estimators can then be used in the pricing equation of the model inorder to be able to obtain the price of a European call option. Further works that can be done in this area include considering the case where these parameters depend on time. The assumption that the interest rate is constant can also be relaxed in future studies.

\section{List of References}

Black F. and Scholes M. (1973). "The Pricing of Options and Corporate Liabilities". Journal of Political Economy 81(3) 637-654.

Brody D, Hughston L. and Macrina A. (2008). "Information-based asset pricing". In- 
ternational Journal of Theoretical and Applied finance 11, 107-142.

Caliskan N.(2007). "Asset Pricing Models:Stochastic Volatility and Information-Based Approaches, Thesis, Department of Financial Mathematics.

Cox C., Ingersoll E. and Ross A. (1985). "A Theory of the Term Structure of Interest Rates". Journal of the Econometric Society 53 (2), 385-407.

Christofiersen, P., Heston, S. and Jacobs, K. (2009). "The shape and term structure of the index option smirk: Why multifactor stochastic volatility models work so well." Management Science, vol. 55, no. 12, 1914-1932.

Da Fonseca J, Grasselli M and Tebaldi C (2007). "Option pricing when correlations are stochastic: An analytical framework". In: Review of Derivatives Research 10, 151-180.

Da Fonseca J, Grasselli M and Tebaldi C (2008). "A multifactor volatility Heston model". In: Quantitative Finance 8, 591-604.

Doucet, A., De Freitas, N., Murphy, K. and Russell, S. (2000). "Rao-blackwellised particle filtering for dynamic bayesian networks. In: Proceedings of the Sixteenth conference on Uncertainty in artificial intelligence," 176-183.

Gatheral, Jim (2006). "The Volatility Surface. A Practitioner's Guide". Wiley Finance.

Hall, A. (2005). Generalized Method of Moments. Advanced Texts in Econometrics, Oxford University Press, New York.

Heston S.L. (1993). "A Closed-Form Solution for Options with Stochastic Volatility with Applications to Bond and Currency Options". In The Review of Financial Studies 6(2) $327-343$.

Higham D.J. (2004). "An Introduction to Financial Option Valuation Mathematics, Stochastics and Computation, Cambridge University Press,Department of Mathematics University of Strathclyde.

Hull, John (2008), "Options, Futures and other Derivatives". Prentice Hall (7), 91-102.

Hull J.C. and White A.(1987), The pricing of options on assets with stochastic volatility, Journal of Finance, 42 281-300.

Julier, S.J. and Uhlmann, J.K. (1996). "A general method for approximating nonlinear transformations of probability distributions." Tech. Rep., Technical Report, RRG,

Lewis, Alan L (2005), "Option Valuation under Stochastic Volatility: With Mathematica Code" Finance Press (2), Newport Beach, 22-57.

Li, J. (2013). "An unscented kalman smoother for volatility extraction: Evidence from stock prices and options." Computational Statistics and Data Analysis, vol. 58, 15-26.

Lu, L., Yang, M., et al. (2015). "Study on importance function for particle filter." International Journal of Multimedia and Ubiquitous Engineering, vol. 10, no. 2, 249258.

Macrina, A. (2006) "An Information-Based Framework for Asset Pricing: X-Factor Theory and its Applications", $\mathrm{PhD}$ Thesis, King's College London.

Maria Cristina Recchioni, M.C. and Scoccia, A. (2014). "An Analytically Tractable Multi-asset Stochastic Volatility Model" In: Applied Mathematical Sciences, 8(27), 1339-1355.

Mikhailow, Sergei and Nögel (2008). "Heston's Stochastic Volatility Model Implementation, Calibration and Some Extensions". Wilmott Magasine.

Merton, Robert C (1973). "Theory of Rational Option Pricing". In: Journal of Economics and Management Science 4(1), 141-183

Mutijah, Guritno, S, and Gunardi, (2012), "A Black Scholes Model from an Information- 
Based Perspective" by Brody Hughston Macrina, International Conference on Statistics in Science, Business and Engineering.

Mutijah, Guritno, S, and Gunardi,(2013), "Estimation of Parameters on the BS-BHM Updated Model." Journal Applied Mathematical Sciences 7(72)

Rouah, F.D. (2013). "The Heston model and its extensions in MATLAB and C\#." John Wiley and Sons.

Rebonato R. (1999), Volatility and Correlation, John Wiley, Chichester, 31-73.

Renault, Eric and Touzi (1996), "Option Hedging and Implied Volatilities in a Stochastic Volatility Model". In: Mathematical Finance 6(3), 279-302.

Shreve S.E. (2004). "Stochastic Calculus for Finance II Continuous-Time Models", Springer. 